\title{
Difference Cesàro sequence space defined by a sequence of modulus function
}

Sunil K. Sharma

Department of mathematics, Cluster University of Jammu, Jammu-180001, J\&K, India.

\begin{abstract}
The purpose of this paper is to introduce the difference sequence space $\operatorname{ces}\left(B_{\Lambda}^{\mu}, F, q\right)$ using sequence of modulus function $F=\left(f_{i}\right)$. We examine some topological properties of the space and also obtain some inclusion relations.
\end{abstract}

Keywords: Cesàro sequence space, difference sequence space, paranormed space.

2020 MSC: 46A45, 40A05, 40C05.

(C)2022 All rights reserved.

\section{Introduction and preliminaries}

Let $w, \ell^{0}$ denote the spaces of all scalar and real sequences, respectively. For $1<p<\infty$, the cesàro sequence space ces $_{p}$ defined by

$$
\operatorname{ces}_{p}=\left\{x \in \ell^{0}: \sum_{n=1}^{\infty}\left(\frac{1}{n} \sum_{k=1}^{n}\left|x_{k}\right|\right)^{p}<\infty\right\},
$$

is a Banach space when equipped with the norm

$$
\|x\|=\left(\sum_{n=1}^{\infty}\left(\frac{1}{n} \sum_{k=1}^{n}\left|x_{k}\right|\right)^{p}\right)^{\frac{1}{p}} .
$$

This space was introduced by Shiue [30], which is useful in the theory of matrix operator. Some geometric properties of the cesàro sequence space $c s_{p}$ were studied by many authors such as Lee [13], Leibowitz [14], Lui et. al [15], Sanhan et. al [25] and Tripathy et. al [33] and references therein. Modulus function has been discussed in [22, 23, 26-29] and references therein.

Ruckle [24] used the idea of a modulus function $f$ to construct a class of FK spaces

$$
L(f)=\left\{x=\left(x_{k}\right): \sum_{k=1}^{\infty} f\left(\left|x_{k}\right|\right)<\infty\right\} .
$$

Email address: sunilksharma42@gmail.com (Sunil K. Sharma)

doi: $10.22436 /$ jnsa.015.01.03

Received: 2021-03-17 Revised: 2021-03-15 Accepted: 2021-05-28 
The space $L(f)$ is closely related to the space $\ell_{1}$ which is an $L(f)$ space with $f(x)=x$ for all real $x \geqslant 0$.

For any set $E$ of sequences, the space of multipliers of $E$, denoted by $M(E)$, is given by

$$
M(E)=\{a \in w: a x \in E, \text { for all } x \in E\} .
$$

The notion of the difference sequence space was introduced by Kızmaz [12]. It was further generalized by Et and Çolak [11] as follows

$$
\mathrm{Z}\left(\Delta^{\mu}\right)=\left\{x=\left(x_{k}\right) \in \omega:\left(\Delta^{\mu} \chi_{k}\right) \in z\right\},
$$

for $z=\ell_{\infty}, c$ and $c_{\circ}$, where $\mu$ is a non-negative integer and

$$
\Delta^{\mu} x_{k}=\Delta^{\mu-1} x_{k}-\Delta^{\mu-1} x_{k+1}, \quad \Delta^{\circ} x_{k}=x_{k}, \quad \forall k \in \mathbb{N},
$$

or equivalent to the following binomial representation

$$
\Delta^{\mu} x_{k}=\sum_{\nu=0}^{\mu}(-1)^{v}\left(\begin{array}{l}
\mu \\
v
\end{array}\right) x_{k+v} .
$$

These sequence spaces were generalized by Et and Basaşir [10] taking $z=\ell_{\infty}(p), c(p)$ and $c_{\circ}(p)$.

Dutta [3] introduced the following difference sequence spaces using a new difference operator:

$$
Z\left(\Delta_{(\eta)}\right)=\left\{x=\left(x_{k}\right) \in \omega: \Delta_{(\eta)} x \in z\right\}, \quad \text { for } z=\ell_{\infty}, c \text { and } c_{\circ},
$$

where $\Delta_{(\eta)} x=\left(\Delta_{(\eta)} x_{k}\right)=\left(x_{k}-x_{k-\eta}\right)$ for all $k, \eta \in \mathbb{N}$.

In [4], Dutta introduced the sequence spaces $\bar{c}\left(\|.,\|,. \Delta_{(\eta)}^{\mu}, p\right), \overline{c_{0}}\left(\|.,\|,. \Delta_{(\eta)}^{\mu}, p\right), \ell_{\infty}\left(\|.,\|,. \Delta_{(\eta)}^{\mu}, p\right)$, $m\left(\|.\|,, \Delta_{(\eta)}^{\mu}, p\right)$, and $m_{\circ}\left(\|.,\|,. \Delta_{(\eta)}^{\mu}, p\right)$, where $\eta, \mu \in \mathbb{N}$ and $\Delta_{(\eta)}^{\mu} x_{k}=\left(\Delta_{(\eta)}^{\mu} x_{k}\right)=\left(\Delta_{(\eta)}^{\mu-1} x_{k}-\Delta_{(\eta)}^{\mu-1} x_{k-\eta}\right)$, and $\Delta_{(\eta)}^{\circ} x_{k}=x_{k}$, for all $k, \eta \in \mathbb{N}$, which is equivalent to the following binomial representation:

$$
\Delta_{(\eta)}^{\mu} x_{k}=\sum_{\nu=0}^{\mu}(-1)^{v}\left(\begin{array}{l}
\mu \\
v
\end{array}\right) x_{k-\eta \nu} .
$$

The difference sequence space have been studied by authors $[5-9,18-21,23,31,32,35]$ and references therein. Başar and Altay [1] introduced the generalized difference matrix $B=\left(b_{m k}\right)$ for all $k, m \in \mathbb{N}$, which is a generalization of $\Delta_{(1)}$-difference operator, by

$$
b_{m k}= \begin{cases}r, & k=m, \\ s, & k=m-1, \\ 0, & (k>m) \text { or }(0 \leqslant k<m-1) .\end{cases}
$$

Başarir and Kayikçi [2] defined the matrix $B^{\mu}\left(b_{m k}^{\mu}\right)$ which reduced the difference matrix $\Delta_{(1)}^{\mu}$ incase $r=$ $1, s=-1$. The generalized $B^{\mu}$-difference operator is equivalent to the following binomial representation:

$$
B^{\mu} x=B^{\mu}\left(x_{k}\right)=\sum_{v=0}^{\mu}\left(\begin{array}{l}
\mu \\
v
\end{array}\right) r^{\mu-v} s^{v} x_{k-v} .
$$

Let $F=\left(f_{i}\right)$ be a sequence of modulus functions, $q=\left(q_{n}\right)$ be a bounded sequence of strictly positive real numbers, then we define the cesàro sequence space as follows

$$
\operatorname{ces}\left(B_{\Lambda}^{\mu}, F, q\right)=\left\{x \in w: \sum_{n=1}^{\infty}\left[f_{i}\left(\frac{1}{n} \sum_{i=1}^{n}\left|B_{\Lambda}^{\mu} x_{i}\right|\right)\right]^{q_{n}}<\infty\right\} .
$$


Taking modulus function $F^{\nu}$ instead of $F$ in the space $\operatorname{ces}\left(B_{\Lambda}^{\mu}, F, q\right)$, we can define the composite space $\operatorname{ces}\left(\mathrm{B}_{\Lambda}^{\mu}, \mathrm{F}^{v}, \mathrm{q}\right)$ as follow

$$
\operatorname{ces}\left(B_{\Lambda}^{\mu}, F^{v}, q\right)=\left\{x \in w: \sum_{n=1}^{\infty}\left[f_{i}^{v}\left(\frac{1}{n} \sum_{i=1}^{n}\left|B_{\Lambda}^{\mu} x_{i}\right|\right)\right]^{q_{n}}<\infty\right\} .
$$

The following inequality will be used throughout the paper. If $0 \leqslant p_{i} \leqslant \sup p_{i}=H, K=\max \left(1,2^{\mathrm{H}-1}\right)$, then

$$
\left|a_{i}+b_{i}\right|^{p_{i}} \leqslant K\left\{\left|a_{i}\right|^{p_{i}}+\left|b_{i}\right|^{p_{i}}\right\},
$$

for all $i$ and $a_{i}, b_{i} \in \mathbb{C}$. Also $|a|^{p_{i}} \leqslant \max \left(1,|a|^{\mathrm{H}}\right)$ for all $a \in \mathbb{C}$.

We examine some topological properties of the space $\operatorname{ces}\left(B_{\Lambda}^{\mu}, F, q\right)$ and also obtain some inclusion relations.

\section{Topological properties}

Theorem 2.1. Let $\mathrm{F}=\left(\mathrm{f}_{\mathrm{i}}\right)$ be a sequence of modulus function and $\mathrm{q}=\left(\mathrm{q}_{\mathrm{n}}\right)$ be a bounded sequence of positive real numbers. Then $\operatorname{ces}\left(\mathrm{B}_{\Lambda}^{\mu}, \mathrm{F}, \mathrm{q}\right)$ is a linear space over the field of complex number $\mathrm{C}$.

Proof. Let $x, y \in \operatorname{ces}\left(B_{\Lambda}^{\mu}, F, q\right)$ and $\alpha, \beta \in \mathbb{C}$. Then there exist positive number $M_{\alpha}$ and $N_{\beta}$ such that $|\alpha| \leqslant M_{\alpha}$ and $|\beta| \leqslant N_{\beta}$. From condition (ii) and (iii) of definition of modulus function and by using inequality (1.1), we have

$$
\begin{aligned}
\sum_{n=1}^{\infty}\left[f_{i}\left(\frac{1}{n} \sum_{i=1}^{\infty}\left|B_{\Lambda}^{\mu}\left(\alpha x_{i}+\beta y_{i}\right)\right|\right)\right]^{q_{n}} \leqslant & \max \left(1,2^{H-1}\right)\left(\max \left(1, M_{\alpha}^{H}\right) \sum_{n=1}^{\infty}\left[f_{i}\left(\frac{1}{n} \sum_{i=1}^{n}\left|B_{\Lambda}^{\mu} x_{i}\right|\right)\right]^{q_{n}}\right. \\
& \left.+\max \left(1, N_{\beta}^{H}\right) \sum_{n=1}^{\infty} \sum_{n=1}^{\infty}\left[f_{i}\left(\frac{1}{n} \sum_{i=1}^{n}\left|B_{\Lambda}^{\mu} y_{i}\right|\right)\right]^{q_{n}}\right) .
\end{aligned}
$$

This implies that $\alpha x+\beta y \in \operatorname{ces}\left(B_{\Lambda}^{\mu}, F, q\right)$. This proves that $\operatorname{ces}\left(B_{\Lambda}^{\mu}, F, q\right)$ is a linear space. This completes the proof of the theorem.

Theorem 2.2. Let $\mathrm{F}=\left(\mathrm{f}_{\mathrm{i}}\right)$ be a sequence of modulus function and $\mathrm{q}=\left(\mathrm{q}_{\mathrm{n}}\right)$ be a bounded sequence of positive real numbers, $\operatorname{ces}\left(\mathrm{B}_{\Lambda}^{\mu}, \mathrm{F}, \mathrm{q}\right)$ is a topological linear space, paranormed by

$$
g(x)=\left(\sum_{n=1}^{\infty}\left[f_{i}\left(\frac{1}{n} \sum_{i=1}^{n}\left|B_{\Lambda}^{\mu} x_{i}\right|\right)\right]^{q_{n}}\right)^{\frac{1}{k}},
$$

where $\mathrm{H}=\sup \mathrm{q}_{\mathrm{n}}<\infty$ and $\mathrm{K}=\max (1, \mathrm{H})$.

Proof. Clearly $g(x)=g(-x)$. It is trivial $B_{\Lambda}^{\mu} x_{\mathfrak{i}}=0$ for $x=0$. Since $f_{i}(0)=0$, we get $g(x)=0$ for $x=0$. Since $\frac{p_{i}}{i} \leqslant 1$, Using the Minkowski's inequality, we have

$$
\begin{aligned}
\left(\sum_{n=1}^{\infty}\left[f_{i}\left(\frac{1}{n} \sum_{i=1}^{\infty}\left|B_{\Lambda}^{\mu}\left(x_{i}+y_{i}\right)\right|\right)\right]^{q_{n}}\right)^{\frac{1}{K}} & \leqslant\left(\sum_{n=1}^{\infty}\left[f_{i}\left(\frac{1}{n} \sum_{i=1}^{\infty}\left(\left|B_{\Lambda}^{\mu} x_{i}\right|+\left|B_{\Lambda}^{\mu} y_{i}\right|\right)\right)\right]^{q_{n}}\right)^{\frac{1}{K}} \\
\leqslant & \left(\sum_{n=1}^{\infty}\left[f_{i}\left(\frac{1}{n} \sum_{i=1}^{\infty}\left|B_{\Lambda}^{\mu} x_{i}\right|\right)\right]^{q_{n}}\right)^{\frac{1}{k}} \\
& +\left(\sum_{n=1}^{\infty}\left[f_{i}\left(\frac{1}{n} \sum_{i=1}^{\infty}\left|B_{\Lambda}^{\mu} y_{i}\right|\right)\right]^{q_{n}}\right)^{\frac{1}{k}}
\end{aligned}
$$


Hence $g(x)$ is subadditive. For the continuity of multiplication, let us take any complex number $\alpha$. By definition, we have

$$
\begin{aligned}
g(\alpha x) & =\left(\sum_{n=1}^{\infty}\left[f_{i}\left(\frac{1}{n} \sum_{i=1}^{\infty}\left|B_{\Lambda}^{\mu}\left(\alpha x_{i}\right)\right|\right)\right]^{q_{n}}\right)^{\frac{1}{K}} \\
& \leqslant C_{\alpha}^{\frac{H}{K}} g(x),
\end{aligned}
$$

where $C_{\alpha}$ is a positive integer such that $|\alpha| \leqslant C_{\alpha}$. Now, let $\alpha \rightarrow 0$ for any fixed $x$ with $g(x) \neq 0$. By definition for $|\alpha|<1$, we have

$$
\sum_{n=1}^{\infty}\left[f_{i}\left(\frac{1}{n} \sum_{k=1}^{\infty}\left|\alpha B_{\Lambda}^{\mu} x_{i}\right|\right)\right]^{q_{n}}<\epsilon, \text { for } n>n_{0}(\epsilon) .
$$

Also, for $1 \leqslant n \leqslant n_{0}$, taking $\alpha$ small enough, since $F=\left(f_{i}\right)$ is continuous, we have

$$
\sum_{n=1}^{\infty}\left[f_{i}\left(\frac{1}{n} \sum_{i=1}^{\infty}\left|\alpha B_{\Lambda}^{\mu} x_{i}\right|\right)\right]^{q_{n}}<\epsilon .
$$

Now, (2.1) and (2.2) together imply that $\mathrm{g}(\alpha \mathrm{x}) \rightarrow 0$ as $\alpha \rightarrow 0$. This completes the proof of the theorem.

Theorem 2.3. Let $\mathrm{F}=\left(\mathrm{f}_{\mathrm{i}}\right)$ be a sequence of modulus function and $\mathrm{q}=\left(\mathrm{q}_{\mathrm{n}}\right)$ be a bounded sequence of positive real numbers, $\operatorname{ces}\left(\mathrm{B}_{\Lambda}^{\mu}, \mathrm{F}, \mathrm{q}\right)$ is a complete paranormed space with paranorm defined by

$$
g(x)=\left(\sum_{n=1}^{\infty}\left[f_{i}\left(\frac{1}{n} \sum_{i=1}^{n}\left|B_{\Lambda}^{\mu} x_{i}\right|\right)\right]^{q_{n}}\right)^{\frac{1}{k}},
$$

where $\mathrm{H}=\sup \mathrm{q}_{\mathrm{n}}<\infty$ and $\mathrm{K}=\max (1, \mathrm{H})$.

Proof. In view of Theorem 2.2 it suffices to prove the completeness of $\operatorname{ces}\left(\mathrm{B}_{\Lambda}^{\mu}, \mathrm{F}, \mathrm{q}\right)$. Let $\left(\chi^{(s)}\right)$ be a Cauchy sequence in $\operatorname{ces}\left(B_{\Lambda}^{\mu}, F, q\right)$. Then $g\left(x^{(s)}-x^{(t)}\right) \rightarrow 0$ as $t \rightarrow \infty$, that is

$$
\sum_{n=1}^{\infty}\left[f_{i}\left(\frac{1}{n} \sum_{i=1}^{\infty}\left|B_{\Lambda}^{\mu}\left(x_{i}^{(s)}-x_{i}^{(t)}\right)\right|\right)\right]^{q_{n}} \rightarrow 0, \quad \text { as } s, t \rightarrow \infty,
$$

which implies that for each $i,\left|x_{i}^{(s)}-x_{i}^{(t)}\right| \rightarrow 0$ as $s, t \rightarrow \infty$ and so $\left(x_{i}^{(s)}\right)$ is a Cauchy sequence in $\mathbb{C}$ for each fixed $i$. Since $\mathbb{C}$ is complete, as $s \rightarrow \infty, x_{i}^{(s)} \rightarrow x_{i}$, for each $i$. Now from (2.3), we have that for $\epsilon>0$, there exists a natural number $\mathrm{N}$ such that

$$
\sum_{n=1}^{\infty}\left[f_{i}\left(\frac{1}{n} \sum_{i=1}^{\infty}\left|B_{\Lambda}^{\mu}\left(x_{i}^{(s)}-x_{i}^{(t)}\right)\right|\right)\right]^{q_{n}}<\epsilon^{K}, \text { for } s, t>N .
$$

Since for any fixed natural number $M$, we have from (2.4)

$$
\sum_{n=1}^{M}\left[f_{i}\left(\frac{1}{n} \sum_{i=1}^{\infty}\left|B_{\Lambda}^{\mu}\left(x_{i}^{(s)}-x_{i}^{(t)}\right)\right|\right)\right]^{q_{n}}<\epsilon^{K}, \text { for } s, t>N,
$$

by taking $t \rightarrow \infty$ in the above expression we obtain

$$
\sum_{n=1}^{M}\left[f_{i}\left(\frac{1}{n} \sum_{i=1}^{\infty}\left|B_{\Lambda}^{\mu}\left(x_{i}^{(s)}-x_{i}\right)\right|\right)\right]^{q_{n}}<\epsilon^{K}, \text { for } s>N .
$$


Since $M$ is arbitrary, by taking $M \rightarrow \infty$, we obtain

$$
\sum_{n=1}^{\infty}\left[f_{i}\left(\frac{1}{n} \sum_{i=1}^{\infty}\left|B_{\Lambda}^{\mu}\left(x_{i}^{(s)}-x_{i}\right)\right|\right)\right]^{q_{n}}<\epsilon^{k}, \text { for } s>N,
$$

i.e., $g\left(x^{s}-x\right)<\epsilon$ for $s>N$. To show that $x \in \operatorname{ces}\left(B_{\Lambda}^{\mu}, F, q\right)$, let $t>M$ and fix $n_{0}$. Since $\frac{p_{i}}{K} \leqslant 1$ and $K \geqslant 1$, using Minkowski's inequality and the definition of modulus function, we have

$$
\begin{aligned}
\left(\sum_{n=1}^{n_{0}}\left[f_{i}\left(\frac{1}{n} \sum_{i=1}^{\infty}\left|B_{\Lambda}^{\mu}\left(x_{i}\right)\right|\right)\right]^{q_{n}}\right)^{\frac{1}{k}} & =\left(\sum_{n=1}^{n_{0}}\left[f_{i}\left(\frac{1}{n} \sum_{i=1}^{\infty}\left|B_{\Lambda}^{\mu}\left(x_{i}-x_{i}^{(t)}+x_{i}^{(t)}\right)\right|\right)\right]^{q_{n}}\right)^{\frac{1}{k}} \\
\leqslant & \left(\sum_{n=1}^{n_{0}}\left[f_{i}\left(\frac{1}{n} \sum_{i=1}^{\infty}\left|B_{\Lambda}^{\mu}\left(x_{i}-x_{i}^{(t)}\right)\right|\right)+f_{i}\left(\frac{1}{n} \sum_{i=1}^{n}\left|B_{\Lambda}^{\mu} x_{i}^{(t)}\right|\right)\right]^{q_{n}}\right)^{\frac{1}{K}} \\
\leqslant & \left(\sum_{n=1}^{n_{0}}\left[f_{i}\left(\frac{1}{n} \sum_{i=1}^{\infty}\left|B_{\Lambda}^{\mu}\left(x_{i}-x_{i}^{(t)}\right)\right|\right)\right]^{q_{n}}\right)^{\frac{1}{K}} \\
& +\left(\sum_{n=1}^{n_{0}}\left[f_{i}\left(\frac{1}{n} \sum_{i=1}^{\infty}\left|B_{\Lambda}^{\mu} x_{i}^{(t)}\right|\right)\right]^{q_{n}}\right)^{\frac{1}{K}} \\
< & \epsilon+g\left(x^{(t)}\right) .
\end{aligned}
$$

It follows that $\sum_{n=1}^{\infty}\left[f_{i}\left(\frac{1}{n} \sum_{i=1}^{n}\left|B_{\Lambda}^{\mu} x_{i}\right|\right)\right]^{q_{n}}$ converges, so that $x=\left(x_{i}\right) \in \operatorname{ces}\left(B_{\Lambda}^{\mu}, F, q\right)$ and the space is complete. This completes the proof of the theorem.

\section{Inclusion relations}

Theorem 3.1. If $q=\left(q_{n}\right)$ and $p=\left(p_{n}\right)$ are bounded sequences of positive real numbers with $0<q_{n} \leqslant p_{n}<\infty$, for each $\mathrm{n}$ and $\mathrm{F}=\left(\mathrm{f}_{\mathrm{i}}\right)$ be a sequence of modulus function, then $\operatorname{ces}\left(\mathrm{B}_{\Lambda}^{\mu}, \mathrm{F}, \mathrm{q}\right) \subseteq \operatorname{ces}\left(\mathrm{B}_{\Lambda}^{\mu}, \mathrm{F}, \mathrm{p}\right)$.

Proof. Let $x \in \operatorname{ces}\left(\mathrm{B}_{\Lambda}^{\mu}, \mathrm{F}, \mathrm{q}\right)$. Then

$$
\sum_{n=1}^{\infty}\left[f_{i}\left(\frac{1}{n} \sum_{i=1}^{n}\left|B_{\Lambda}^{\mu} x_{i}\right|\right)\right]^{q_{n}}<\infty
$$

This implies that $f_{i}\left(\frac{1}{n} \sum_{i=1}^{n}\left|B_{\Lambda}^{\mu} x_{i}\right|\right) \leqslant 1$ for sufficiently large values of $n$, say $n \geqslant n_{0}$ for some fixed $n_{0} \in \mathbb{N}$. Since $F=\left(f_{i}\right)$ is increasing and $q_{n} \leqslant p_{n}$, we have

$$
\sum_{n \geqslant n_{0}}^{\infty}\left[f_{i}\left(\frac{1}{n} \sum_{i=1}^{n}\left|B_{\Lambda}^{\mu} x_{i}\right|\right)\right]^{p_{n}} \leqslant \sum_{n \geqslant n_{0}}^{\infty}\left[f_{i}\left(\frac{1}{n} \sum_{i=1}^{n}\left|B_{\Lambda}^{\mu} x_{i}\right|\right)\right]^{q_{n}}<\infty,
$$

which implies that $x \in \operatorname{ces}\left(B_{\Lambda}^{\mu}, F, p\right)$ and this completes the proof of the theorem.

Theorem 3.2. If $\mathrm{u}=\left(\mathrm{u}_{\mathrm{n}}\right)$ and $v=\left(v_{\mathrm{n}}\right)$ are bounded sequences of positive real numbers with $0<\mathrm{u}_{\mathrm{n}}, v_{\mathrm{n}}<\infty$, and $\mathrm{q}_{\mathrm{n}}=\min \left(\mathrm{u}_{\mathrm{n}}, v_{\mathrm{n}}\right)$, then

$$
\operatorname{ces}\left(B_{\Lambda}^{\mu}, F, q\right)=\operatorname{ces}\left(B_{\Lambda}^{\mu}, F, u\right) \cap \operatorname{ces}\left(B_{\Lambda}^{\mu}, F, \nu\right) .
$$

Proof. It follows from Theorem 3.1 that

$$
\operatorname{ces}\left(B_{\Lambda}^{\mu}, F, q\right) \subset \operatorname{ces}\left(B_{\Lambda}^{\mu}, F, u\right) \cap \operatorname{ces}\left(B_{\Lambda}^{\mu}, F, v\right) .
$$

For any complex number $\lambda,|\lambda|^{\mathfrak{q}_{n}} \leqslant \max \left(|\lambda|^{\mathfrak{u}_{n}},|\lambda|^{v_{n}}\right)$, thus

$$
\operatorname{ces}\left(B_{\Lambda}^{\mu}, F, u\right) \cap \operatorname{ces}\left(B_{\Lambda}^{\mu}, F, \nu\right) \subseteq \operatorname{ces}\left(B_{\Lambda}^{\mu}, F, q\right),
$$

and the proof of the theorem is complete. 
Theorem 3.3. If $\mathrm{H}=\sup \mathrm{p}_{\mathrm{k}}<\infty$ and $\mathrm{F}=\left(\mathrm{f}_{\mathrm{i}}\right)$ be a sequence of modulus function, then $\ell_{\infty} \subset \mathrm{M}\left(\operatorname{ces}\left(\mathrm{B}_{\wedge}^{\mu}, \mathrm{F}, \mathrm{q}\right)\right)$. Proof. $a \in \ell_{\infty}$ implies $\left|a_{i}\right|<1+[i]$ for some $i>0$ and all $i$. Hence, $x \in \operatorname{ces}\left(B_{\Lambda}^{\mu}, F, q\right)$ implies

$$
\sum_{n=1}^{\infty}\left[f_{i}\left(\frac{1}{n} \sum_{i=1}^{n}\left|a_{i} x_{i}\right|\right)\right]^{a_{n}}<(1+[i])^{H} \sum_{n=1}^{\infty}\left[f_{i}\left(\frac{1}{n} \sum_{i=1}^{n}\left|B_{\Lambda}^{\mu} x_{i}\right|\right)\right]^{q_{n}},
$$

which gives $\ell_{\infty} \subset M\left(\operatorname{ces}\left(B_{\Lambda}^{\mu}, F, q\right)\right)$. This completes the proof of the theorem.

Theorem 3.4. For any sequence of modulus function $\mathrm{F}=\left(\mathrm{f}_{i}\right)$ and $v \in \mathbb{N}$,

(i) $\operatorname{ces}\left(B_{\Lambda}^{\mu}, F^{v}, q\right) \subseteq \operatorname{ces}\left(B_{\Lambda}^{\mu}, q\right)$, if $\lim _{t \rightarrow \infty} \frac{f(t)}{t}=\beta>0$.

(ii) $\operatorname{ces}\left(\mathrm{B}_{\Lambda}^{\mu}, \mathrm{q}\right) \subseteq \operatorname{ces}\left(\mathrm{B}_{\Lambda}^{\mu}, \mathrm{f}^{v}, \mathrm{q}\right)$, if there exists a positive constants $\alpha$ such that $\mathrm{f}(\mathrm{t}) \leqslant \alpha \mathrm{t}$, for all $\mathrm{t} \geqslant 0$.

Proof. (i) By Maddox [12, Proposition 1], we have

$$
\beta=\lim _{t \rightarrow \infty} \frac{f(t)}{t}=\inf \left\{\frac{f(t)}{t}: t>0\right\},
$$

so that $0 \leqslant \beta \leqslant f(1)$. Let $\beta>0$, by definition of $\beta$, we have $\beta t \leqslant f(t), \forall t \geqslant 0$. Since $F=\left(f_{i}\right)$ is increasing we have $\beta^{2} t \leqslant f^{2}(t)$. So by induction we have $\beta^{v} t \leqslant f^{v}(t)$. Let $x \in \operatorname{ces}\left(F^{v}, q, B_{\Lambda}^{\mu}\right)$, Using inequality $|\lambda|^{q_{i}} \leqslant \max \left(1,|\lambda|^{\mathrm{H}}\right)$, we have

$$
\begin{aligned}
\sum_{n=1}^{\infty}\left(\frac{1}{n} \sum_{i=1}^{n}\left|B_{\Lambda}^{\mu} x_{i}\right|\right)^{q_{n}} & \leqslant \sum_{n=1}^{\infty}\left[\beta^{-v} f_{i}^{\nu}\left(\frac{1}{n} \sum_{i=1}^{n}\left|B_{\Lambda}^{\mu} x_{i}\right|\right)\right]^{q_{n}} \\
& \leqslant \max \left(1, \beta^{-v H}\right) \sum_{n=1}^{\infty}\left[f_{k}^{v}\left(\frac{1}{n} \sum_{i=1}^{n}\left|B_{\Lambda}^{\mu} x_{i}\right|\right)\right]^{q_{n}},
\end{aligned}
$$

and hence $x \in \operatorname{ces}\left(B_{\Lambda}^{\mu}, q\right)$.

(ii) Since $f_{i}(t) \leqslant \alpha t$, for all $t \geqslant 0$ and $F=\left(f_{i}\right)$ is an increasing function, we have $f_{i}^{v}(t) \leqslant \alpha^{v} t$ for each $v \in \mathbb{N}$. Let $x \in \operatorname{ces}\left(B_{\Lambda}^{\mu}, q\right)$. Using inequality $|\lambda|^{q_{i}} \leqslant \max \left(1,|\lambda|^{H}\right)$, we have

$$
\sum_{n=1}^{\infty}\left[f_{i}^{\nu}\left(\frac{1}{n} \sum_{i=1}^{n}\left|B_{\Lambda}^{\mu} x_{i}\right|\right)\right]^{q_{n}} \leqslant \max \left(1, \alpha^{\nu H} \sum_{n=1}^{\infty}\left(\frac{1}{n} \sum_{i=1}^{n}\left|B_{\Lambda}^{\mu} x_{i}\right|\right)^{q_{n}},\right.
$$

and hence $x \in \operatorname{ces}\left(\mathrm{B}_{\Lambda}^{\mu}, \mathrm{F}^{\nu}, \mathrm{q}\right)$.

Theorem 3.5. Let $\mathrm{m}, v \in \mathbb{N}$ be such that $\mathrm{m}<v$. If there exists a positive constant $\alpha$ such that $\mathrm{f}(\mathrm{t}) \leqslant \alpha \mathrm{t}$ for all $t \geqslant 0$, then

$$
\operatorname{ces}\left(B_{\Lambda}^{\mu}, q\right) \subseteq \operatorname{ces}\left(B_{\Lambda}^{\mu}, F^{m}, q\right) \subseteq \operatorname{ces}\left(B_{\Lambda}^{\mu}, F^{v}, q\right)
$$

Proof. Let $r=v-m$. Since $f_{i}(t) \leqslant \alpha t$, we have $f^{v}(t)<M^{r} f_{k}^{m}(t)<M^{v} t$, where $M=1+[\alpha]$. Let $x \in \operatorname{ces}\left(B_{\Lambda}^{\mu}, q\right)$, we have

$$
\begin{aligned}
\sum_{n=1}^{\infty}\left[f_{k}^{\nu}\left(\frac{1}{n} \sum_{i=1}^{n}\left|B_{\Lambda}^{\mu} x_{i}\right|\right)\right]^{q_{n}} & <M^{r H} \sum_{n=1}^{\infty}\left[f_{i}^{m}\left(\frac{1}{n} \sum_{i=1}^{n}\left|B_{\Lambda}^{\mu} x_{i}\right|\right)\right]^{q_{n}} \\
& <M^{v H} \sum_{n=1}^{\infty}\left(\frac{1}{n} \sum_{i=1}^{n}\left|B_{\Lambda}^{\mu} x_{i}\right|\right)^{q_{n}},
\end{aligned}
$$

and the required inclusion follows. This completes the proof.

\section{Acknowledgment}

I would like to thanks reviewers for their valuable suggestions and comments which improve the presentation of the paper. 


\section{References}

[1] F. Başar, B. Altay, On the space of sequences of $\mathrm{p}$-bounded variation and related matrix mappings, Ukrainian Math. J., 55 (2003), 136-147. 1

[2] M. Başarir, M. Kayikçi, On the generalized $\mathrm{B}^{\mathrm{m}}$-Riesz difference sequence spaces and $\beta$-property, J. Inequal. Appl., 2009 (2009), 18 pages. 1

[3] H. Dutta, On some difference sequence spaces, Pacific J. Sci. Tech., 10 (2009), 243-247. 1

[4] H. Dutta, Some statistically convergent difference sequence spaces defined over real 2-normed linear space, Appl. Sci., 12 (2010), 37-47. 1

[5] A. Esi, Generalized difference sequence spaces defined by Orlicz functions, Gen. Math., 17 (2009), 53-66. 1

[6] A. Esi, Some classes of generalized difference paranormed sequence spaces associated with multiplier sequences, J. Comput. Anal. Appl., 11 (2009), 536-545.

[7] A. Esi, M. Işik, Some generalized difference sequence spaces, Thai J. Math., 3 (2005), 241-247.

[8] A. Esi, B. C. Tripathy, Strongly almost convergent generalized difference sequences associated with multiplier sequences, Math. Slovaca, 57 (2007), 339-348.

[9] A. Esi, B. C. Tripathy, B. Sarma, On some new type generalized difference sequence spaces, Math. Slovaca, 57 (2007), 475-482. 1

[10] M. Et, M. Basarir, On some new generalized difference sequence spaces, Period. Math. Hungar., 35 (1997), 169-175. 1

[11] M. Et, R. Çolak, On some generalized difference sequence spaces, Soochow J. Math., 21 (1995), 377-356. 1

[12] H. Kizmaz, On certain sequences spaces, Canad. Math. Bull., 24 (1981), 169-176. 1, 3

[13] P. Y. Lee, Cesàro sequence spaces, Math. Chronicle, 13 (1984), 29-45. 1

[14] G. M. Leibowitz, A note on the Cesàro sequence spaces, Tamkang J. Math., 2 (1971), 151-157. 1

[15] Y. Q. Lui, B. E. Wu, P. Y. Lee, Method of sequence spaces, Guangdong of Science and Technology Press, China, (1996). 1

[16] I. J. Maddox, Sequence spaces defined by a modulus, Math. Proc. Cambridge Philos. Soc., 100 (1986), 161-166.

[17] I. J. Maddox, Inclusion between FK spaces and Kuttner's theorem, Math. Proc. Cambridge Philos. Soc., 101 (1987), 523-527.

[18] S. A. Mohiuddine, K. Raj, M. Mursaleen, A. Alotaibi, Linear isomorphic spaces of fractional order difference operators, Alexandria Eng. J., 60 (2021), 1155-1164. 1

[19] M. Mursaleen, A. Alotaibi, S. K. Sharma, Some new lacunary strong convergent vector-valued sequence spaces, Abstr. Appl. Anal., 2014 (2014), 8 pages.

[20] M. Mursaleen, S. K. Sharma, Entire sequence spaces defined on locally convex Hausdorff topological space, Iran. J. Sci. Technol. Trans. A Sci., 38 (2014), 105-109.

[21] M. Mursaleen, S. K. Sharma, S. A. Mohiuddine, A. Kiliçman, New difference sequence spaces defined by MusielakOrlicz function, Abstr. Appl. Anal., 2014 (2014), 9 pages. 1

[22] K. Raj, S. A. Mohiuddine, M. Mursaleen, Some generalized sequence spaces of invariant means defined by ideal and modulus functions in n-normed spaces, Italian J. Pure Appl. Math., 39 (2018), 579-595. 1

[23] K. Raj, S. K. Sharma, Difference sequence spaces defined by sequence of modulus function, Proyecciones, 30 (2011), 189-199. 1

[24] W. H. Ruckle, FK spaces in which the sequence of coordinate vectors is bounded, Canadian J. Math., 25 (1973), 973-978. 1

[25] W. Sanhan, S. Suantai, On k-nearly uniform convex property in generalized Cesàro sequence spaces, Int. J. Math. Math. Sci., 57 (2003), 3599-3607. 1

[26] E. Savas, On some generalized sequence spaces defined by a modulus, Indian J. pure Appl. Math., 30 (1999), 459-464. 1

[27] S. K. Sharma, Generalized sequence spaces defined by a sequence of moduli, J. Egyptian Math. Soc., 23 (2015), $73-77$.

[28] S. K. Sharma, A. Esi, Some I-convergent sequence spaces defined by using sequence of moduli and n-normed space, J. Egyptian Math. Soc., 21 (2013), 103-107.

[29] S. K. Sharma, A. Esi, Double sequence spaces defined by a sequence of modulus function over n-normed space, Acta Univ. Palack. Olomuc. Fac. Rerum Natur. Math., 53 (2014), 117-134. 1

[30] J.-S. Shiue, On the Cesaro sequence spaces, Tamkang J. Math., 1 (1970), 19-25. 1

[31] B. C. Tripathy, A. Esi, Generalized lacunary difference sequence spaces defined by Orlicz functions, Matimyas Mat., 28 (2005), 50-57. 1

[32] B. C. Tripathy, A. Esi, A new type of difference sequence spaces, Int. J. Sci. Tech., 1 (2006), 11-14. 1

[33] B. C. Tripathy, A. Esi, B. Tripathy, On a new type of generalized difference Cesáro sequence spaces, Soochow J. Math., 31 (2005), 333-340. 1

[34] A. Wilansky, Summability through functional analysis, North-Holland Publishing Co., Amsterdam, (1984).

[35] T. Yaying, B. Hazarika, S. A. Mohiuddine, M. Mursaleen, K. J. Ansari, Sequence spaces derived by the triple band generalized Fibonacci difference operator, Adv. Difference Equ., 2020 (2020), 19 pages. 1 